\title{
DEVELOPMENT OF APPROACHES TO A NEW TYPE OF ANTITHROMBOTIC DRUGS BASED ON SULFUR-CONTAINING MONOTERPENOIDS
}

\author{
L.E. Nikitina ${ }^{1,2}$, S.V. Kiselev', Z.R. Azizova', R.S. Pavelyev', I.R. Gilfanov', \\ A.A. Ksenofontov ${ }^{3}$, P.S. Bocharov ${ }^{3}$, E.V. Antina ${ }^{3}$, O.V. Ostolopovskaya ${ }^{2}$, \\ M.A. Khelkhal ${ }^{2}$, V.V. Klochkov ${ }^{2}$ and A.R. Kayumov ${ }^{2}$
}
${ }^{1}$ Kazan State Medical University, 420012, Russia, Kazan, Butlerova str., 49.
${ }^{2}$ Kazan Federal University, 420008, Russia, Kazan, Kremlevskaya str., 18.
${ }^{3}$ G.A. Krestov Institute of Solution Chemistry of Russian Academy of Sciences, 153045, Russia, Ivanovo, Akademicheskaya str., 1.

DOI: 10.19163/MedChemRussia2021-2021-144

E-mail: nikitl@mail.ru

The blood coagulation is known reason of various diseases and the development of novel anticoagulants is the challenge of modern pharmacy. The results of our previous investigations of antiplatelet and anticoagulative activity of sulfur-containing monoterpenoids found out promising possibility of their using as potential antithrombotics [1,2]. Here we report the effect of newly synthesized $\mathrm{S}, \mathrm{N}, \mathrm{O}$-containing monoterpenoids on blood coagulation in vitro. While all tested compounds exhibited antiplatelet properties, the most pronounced effect was observed for the S-containing compound. Molecular docking revealed the putative interaction of the platelet's $\mathrm{P}_{2} \mathrm{Y}_{12}$ receptor with all tested compounds suggesting that antiplatelet properties is apparently implemented by blocking the receptor activity of platelets. The calculated binding energy depended on heteroatom and significantly decreased with changing the sulfur atom to oxygen or nitrogen ones.

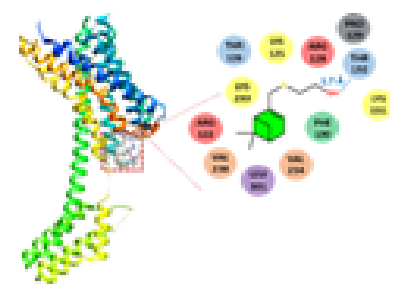

Furthermore, in NMR studies using dodecylphosphocholine (DPC) as membrane model only S-containing compound was found to be bounded with DPC micelles surface, while no stable complexes between DPC micelles with either 0- or $\mathrm{N}$-containing compounds were observed. Apparently, the binding of S-containing compound with cellular membrane reinforces mechanical properties of the latter thereby preventing its destabilization and following clot formation on phospholipid surface. In general, our data demonstrate that S-containing compounds exhibit antiplatelet properties via both membrane stabilization and blocking $\mathrm{P}_{2} \mathrm{Y}_{12}$ receptor and thus appears as promising anticoagulant for the hemostasis control.

This work was supported by the Russian Science Foundation (project 20-64-47014).

\section{References}

[1] Nikitina, L. E.; Kiselev, S. V; Startseva, V. A. et al., Front. Pharmacol.2018, 9, 116.

[2] Liliya E. Nikitina, Roman S. Pavelyev, Valeriya A. Startseva et al., J. Molecular Liquids. 2020, 301. Art. №: 112366. 